\title{
Sense-making with Inscriptions in Quantum Mechanics
}

\author{
Erin Ronayne Sohr, Ayush Gupta, Andrew Elby, Benjamin W. Dreyfus \\ University of Maryland, Department of Physics \\ College Park, Maryland, 20742
}

\begin{abstract}
We focus on a student Chad's sense-making with representations (inscriptions) of both standard forms found in quantum textbooks and emergently constructed non-standard forms. Through fine-timescale analysis of video from a clinical interview, we document the different ways in which the inscription becomes embedded in the student's reasoning. Our findings show that the inscriptional system generated by Chad shapes, and is shaped by, his sense-making and causal reasoning, and that the standard forms recruited can provide a space for action, beyond that of simple read-out. We briefly touch on some guiding instructional design choices and directions of future research.
\end{abstract}

\section{INTRODUCTION}

Developing proficiency with representations such as graphs, equations, schematics, diagrams, and free-body diagrams, though central to learning and problem-solving in physics [1-5], is often challenging for students [6,7]. Our goal is not to solve these difficult instructional issues, but rather, to problematize what counts as proficiency. Typically, research on instructional interventions deems students proficient with a representation if students can demonstrate the skills of correctly generating the representation and extracting information (reading out) from the representation. Recent research, however, has started to build a more nuanced story of students' use of representations during sense-making, going beyond a "skills development" narrative. For example, Heckler (2015) shows that requiring students to draw diagrams can produce a disconnect between the diagram and the student's sense-making [8]. Students who construct the diagram unprompted tend to do better at integrating the diagram with conceptual reasoning. Others, for example Gire et al. [9] and Parnafes [10], have shown that different physical features within representations may serve different roles in student reasoning, providing support for computation or for conceptual ideas such as "fast." The picture that emerges from these studies is that representation use by physics learners is contextual and tied closely with their conceptual reasoning.

In this paper, we extend the thread of research on the dynamics of students' sense-making and inscription use. We draw on Greeno \& Hall's [11] framework for describing representational practices students should engage in, which we term flexible use for brevity. Flexible use is characterized in part by having representation(s) play an integral role in communication and problem-solving.

To study flexible use, we "tweaked" the traditional particle in a box scenario in introductory quantum physics to see how students would use inscriptions when sensemaking in this novel context [7]. Here, we do a microanalysis [12] of a single episode from our interview with a student, pseudonymed Chad. Chad, we argue, creates a rich system of inscriptions to serve various coordinating, communicating, and reasoning purposes. Chad generates and uses the inscriptions reflexively, in the sense that his con- ceptual thinking and his inscriptions change in ways that mutually affect each other. Our goal with this preliminary work is to begin to describe and characterize reflexive use of inscriptions, which in this case, challenges traditional notions of proficiency in using representations. These preliminary results will inform future work on the analysis of student reasoning with inscriptions, as well as instructional design of representation tasks.

\section{FLEXIBLE USE OF INSCRIPTIONS}

Greeno \& Hall [11] argue for instruction that provides opportunities to flexibly and creatively participate in the practices of representation, and against those that teach representations solely as end-goals. In flexible use, students participate in inventing and refining the form and interpretation of representations. The authors particularly advocate allowing students the opportunity to construct and adapt forms, both standard and non-standard, as tools for planned and emergent sense-making and communication needs.

Within flexible use, student sense-making with inscriptions can take on a reflexive character, potentially arising from the different affordances of different forms [2, 11]. Greeno \& Hall describe such interaction as "[a] process in which students construct representations based on partial understanding and then can use the representations to improve their understanding, which leads to a more refined representation, and so on." Practically, others such as Lehrer et al. [13] have used case studies to track the evolution of inscriptions of children to show how inscriptions "govern the evolution of children's thinking." In this paper, we analyze the sense-making of an individual who demonstrates this kind of reflexive interplay between his sensemaking and the figural form of his inscriptions.

\section{METHODOLOGY}

Greeno \& Hall's framework [11] helped guide the design of our interview protocol. Some of these principles (generating problems with ill-defined end-points, which invite multiple solutions and have a focus on functionality) are suggested to be part of good design problems, as well as reflections of disciplinarily authentic problems [13]. As, such aimed to provide students ample opportunity to con- 
struct forms, adapt standard forms, participate in conventions of interpretation, and use their forms to communicate their sense-making to the interviewer (ERS). Design choices included: problems where students were likely to adapt standard forms (e.g., ground state of a particle in a box with a slanted "floor"), epistemological re-framings (explaining a quantum system to another student), conceptual reframings (finding the "wavefunction" for a classical particle), and evaluations of different disciplinary forms (stacked versus separate representations of wavefunctions).

Our interviewees were upper-division physics and engineering students working through collections of tutorialstyle problems. During content logging of the video-taped interviews, ERS noted what appeared to be hints of common student difficulties associated with use of representations in quantum mechanics [6, 7]. However, upon closer and collective viewing [14], the authors found that the patterns of student reasoning were not fully captured by the student difficulties reported in the literature $[6,7]$.

We iteratively moved between individual and collective analysis of the data [14]. With each analytical pass, we drew on interaction analysis [14] to attend to the content and paralinguistic features of the student and interviewer's discourse, such as gaze, posture, and gestures. Interaction analysis provided the tools to identify relevant entities in the students' sense-making. Microgenetic learning analysis [12] provided additional fine-grained meaning to our interaction analysis, helping us to distinguish subtle changes in and differences between conceptual and inscription-based reasoning. In analyzing the data, we sought to answer the two questions: How does the figural form shape the content (conceptual or otherwise) of Chad's sense-making? How does Chad's sense-making (conceptual or otherwise) shape the inscriptional system?

The interview episode analyzed here comes from a senior physics major, "Chad," who had finished a twosemester quantum sequence the previous semester. We chose a three-minute episode that highlighted the rich nature of Chad's reasoning and inscription use. In the episode, Chad addresses the question, "Consider a quantum system with $V(x)=\infty$ for $x=(-\infty, 0)$ and $(L, \infty)$, and $V(x)=A x$ for $x=(0, L)$. Sketch the wavefunction for the first allowed state, or ground state, of the particle."

\section{RESULTS}

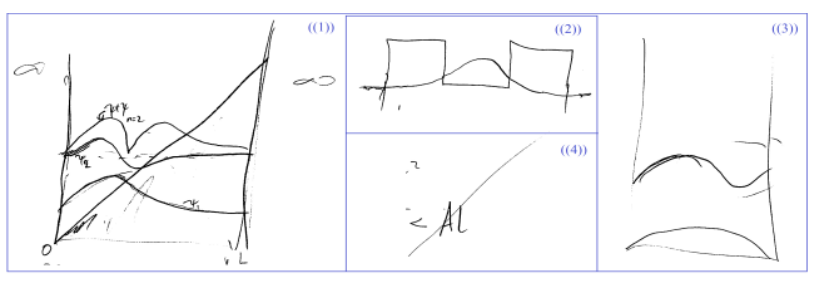

FIG. 1. Chad's inscriptions, labeled ((1))-((4)) clockwise.

Our analysis shows a reflexive interplay between the conceptual ideas in Chad's sense-making and the physical form of the inscriptions he creates. Within such reflexive use, he creates a non-standard form through the coordination and manipulation of two standard forms, that of a finite potential well and the stacked-wavefunction PIAB representation (diagrams $((2))$ and $((3))^{1}$ in Fig. 1 above).

${ }^{2}$ After reading the problem, Chad spends a few turns establishing the problem space. He starts drawing the standard form of particle in a box (PIAB), but soon realizes the potential is different, and proceeds to erase and slant the bottom of the well. He mentions that drawing the allowed states was a final exam question in his classes. As of now, Chad has drawn the slanted PIAB potential well, without any wavefunctions inside. He begins to discuss the problem, and seems to find use for a new inscription to facilitate and support his sense-making. Chad continues with:

Chad: We talk about how the potential walls affect it ((traces vertical wall on 1$))$. And how it would be uhh... ((re-traces vertical wall on 1)) Yeah 'cus we talked, yeah if you talk about, uhh finite regions ((draws 2)), you have the wavefunction in here ((begins to draw wavefunction in 2)), it doesn't go to zero here ((crosses boundary in 2)), it goes to the points that it does, then exponentially decays in it ((draws decaying wavefunction in left, then right region)).

At the beginning of his previous turn, Chad has a platform ready $((1))$ to hold his representation of the "states allowed." Chad begins a chain of causal reasoning [15] in stating that the "potential walls affect it." In doing so, he highlights and re-highlights the vertical, right line in ((1)), bringing forth one aspect of the inscription as particularly relevant to the shared visual field [16]. The overlapping speech and tracing indicate that Chad is referring to the vertical line as a "potential wall." As the episode progresses, we see continued evidence that the regions and boundaries created by walls in his inscriptions structure and coordinate his reasoning.

After an abandoned start, "and how it would be...," Chad moves vertically down his page and begins to draw ((2)), a standard representation of a finite potential well. The inscription ((2)) unfolds with his speech; he notes that the wavefunction "exponentially decays within it." The use of the preposition "within" and his concurrent drawing of the wavefunctions in the regions of high potential connects the idea of "decay" to regions bounded by potential walls in the inscription.

In this short piece of speech, the physical structure of the potential walls feeds into his sense-making in several ways. Looking at his utterance, once he begins to expand on his causal reasoning of how the "potential walls affect it," his speech is naturally punctuated at the potential wall

\footnotetext{
${ }^{1}$ In transcripts and in our description, numbers included within double parentheses $(())$ references written diagrams, and the text within $(())$ references Chad's actions or gestures on referenced diagrams.

${ }^{2}$ Full transcript and larger versions of written work can be found here: http://hdl.handle.net/1903/18472
} 
boundaries. The clauses of his speech either refer to a region of the inscription ("uhh finite regions," "you have the wavefunction in here," and "then exponentially decays in it") or refer to the values at boundaries in the inscription ("it doesn't go to zero here" and "it goes to the points that it does"). His reasoning about the wavefunction is compartmentalized into reasoning about its properties in regions and at boundaries [15]. We therefore infer that the representation is playing some role in 'bounding' Chad's reasoning, perhaps by supporting a causal or descriptive account he is remembering, or partially generating on the fly, about wavefunction behavior.

Following Chad's utterance, the interviewer acknowledges Chad's contribution, and he continues with:

Chad: and then... if they're tall enough, you get ((extends boundary walls down 2))... tunneling ((extends wavefunction horizontally in 2))!

Chad takes the outermost walls of the inscription and extends them further down. When he adds to the wavefunction, he draws the piece of the wavefunction that extends horizontally beyond the boundary walls that he had just previously extended. In this brief turn, Chad recounts a causal story about the emergence of tunneling, which can occur if these pieces of the wavefunction are "tall enough;" a condition which does not have empirical conceptual underpinnings, but is more readily a condition met by the physical form of the inscription. The first form of the inscription (without extended walls and wavefunction), provides the set-up conditions necessary for the tunneling to occur [15]. To be clear, the causal story of tunneling is told more through the manipulation of the physical form of the inscription than readily apparent conceptual ideas about the particle or probability. As before, the region-based structure of the inscription structures Chad's speech in his implicit treatment of the wavefunction as piece-wise, through his coding of these pieces of the wavefunction as "they" [16], where "they" end at the boundaries of potential walls.

Chad now turns back to the platform for wavefunctions he created with $((1))$ and begins to fill in the first two allowed states. He says:

Chad: but yeah for this one it would just be, uhh it starts off like it and then it decays ((draws lowest state as he speaks, draws higher state silently)).

At this point, the thread about tunneling has died away and Chad instead focuses on the physical shape of the lowest wavefunction. On being asked, Chad further elaborates how he drew the wavefunctions, generating new and standard inscriptions along the way:

Chad: So it's, you can kind of take it as perturbation upon the particle in a box ((draws box in 3)). So it's going to be essentially particle in a box ((draws first two states in 3)) but then, uhh what's happening is as the potential increases ((draws 4$))$, it reduces the probability of being in that re- gion. Which means that if you still normalize it ((traces higher state in 3)), it would have to follow, it would have to follow the same energy, stepping, where it's going by nodes added, but it will reduce the probability of this region ((circles right side of higher state in 3)), linearly.

The new conceptual ideas emerging are that the problem is essentially a perturbation on the usual PIAB, and the potential and probability are inversely related. Again, it's important to note that the relationship between potential and probability is coordinated through reference to given "regions," now referring to the higher-potential vs. lowerpotential parts of the interior of the "box." The potentialprobability relationship he posited is also continuous with, and in some ways a refinement of, his previous conclusion that the potential walls have an effect on the wavefunction. Chad's verbal coordination of these two conceptual ideas leads to the creation and manipulation of new inscriptions. The inscriptional system grows to include a standard form of a PIAB representation $((3))$ with a slanted line $((4))$ positioned directly below $((3))$. His reasoning appears to structure and organize the construction of the inscriptions through this vertical alignment: Chad can more easily readout the locations of areas of high potential, and therefore high effect on probability. Chad's speech initially sets-up the mediating relationship between two conceptual ideas. However, it's the strong visual coordination between inscriptions ((3)) and ((4)) that provides the platform for reasoning and showing that the right side of the wavefunction in $((3))$ will see reduced "probability of being in that region." He circles the region of interest in $((3))$, the interviewer then confirms that he is implicitly also reasoning about the corresponding region in $((1))$, to which he agrees. Ultimately, through this interaction, Chad manipulates the standard form of ((3)), through the mediating effect of $((4))$, in order to draw conclusions about reduced probability in the right side of the ground state in the slanted PIAB ((1)).

Chad continues his reasoning about the shapes of the wavefunctions in the slanted PIAB:

Chad: but, so it will follow essentially it ((traces lower state in 1))... I think I made it too big for my waves to look right. But it will go into it generally like that ((points to lower state in 3)). But it will also decay ((traces right side of lower state in 1)) after it enters the region.

He traces the wavefunction as he attends to the deviation from the unperturbed PIAB, leaving a little line that the normal ground state might follow, where the slanted ground state begins to decay away.

In this concluding bit of speech, Chad finalizes his conceptual coordination of the unperturbed PIAB and the mediating effect of the potential increasing. He turns to the slanted PIAB, and shows how the state takes on characteristics of the unperturbed PIAB and then begins to decay towards the right side of the inscription. Although interpretations are possible whereby the various representations 
merely express Chad's thinking, we argue for a more reflexive relation between the inscriptions and Chad's thinking whereby the inscriptions influence and help shape his thinking: the decaying wavefunctions inscribed in the forbidden regions of ((2)), with forbidden corresponding visually to where the potential is higher than the wavefunction, combined with the visual coordination of ((3)) and ((4)) as discussed above, contributes to Chad's in-the-moment drawing/thinking about the lowest wavefunction in ((1)). The particular shape of the wavefunction - unperturbed then decaying - as well as the conceptual insight that the probability is lower where the potential is higher are constructed through the generation, manipulation, and reasoning with the inscriptions in $((2,3,4))$.

\section{DISCUSSION}

In this brief episode, Chad's sense-making and inscription use are deeply entwined, occasionally unfolding together and other times feeding back into each other reflexively. In some moments, his talk was structured in terms of the properties of regions of the inscription. In some places, Chad's judicious use of inscriptions may provide additional structure to the conceptual ideas he brings up. For example, his speech explicitly relays that the problem is like the standard PIAB, but that there is some mediating effect of the potential increasing. However, the cognitive work to find the region affected in ((1)), where the wavefunction is "below" the potential, is not accomplished in his speech, but through the coordination of other inscriptions.

For Chad, different inscriptions do different work, and he generates new inscriptions to serve specific, emergent purposes [11]; even the standard representations he draws do not simply serve read-out purposes. Chad does work on these inscriptions to coordinate different conceptual ideas, giving additional meaning to the non-standard form he constructs, ((1)). Not only do different inscriptions serve different purposes for Chad, different parts of individual inscriptions play different roles. For example, the right side of ((1)) initiates the causal reasoning about how the rising potential affects the probability, with the regions of wavefunction decay in ((2)) playing a role in that reasoning.

\section{CONCLUSIONS AND IMPLICATIONS}

In this paper, we began with Greeno \& Hall's [11] invocation for teachers to create opportunities for students to use representations flexibly. Towards this purpose, we described some design principles used in problem development and recounted an episode of student reasoning in the context of the designed problems that illustrates flexible, reflexive use of representations.

We suspect that the coordinating role of the interviewer, the norms associated with the interview space as a place to sense-make, among other things, create an interactional space that helps this type of reasoning unfold. This suggests that, as instructors, providing opportunities for flexible use of representations might involve creating an instructional environment for sense-making. However, our goal is not to fully prescribe instructional design principles. We encourage instructors to turn to the publications [11, 13] from which we draw influence for a more complete account of authentic means of using representations in classrooms [11] and some of the benefits of good design problems [13].

Our goal is to expand upon the notion of reflexive use of representation through our case-study of Chad, showing the coupled, mutually influencing nature of Chad's sensemaking and his developing inscriptional system. Through this case-study, we hope to begin challenging what counts as proficiency in using representations. Reflexive use describes a pattern of sense-making that may involve a backand-forth between the traditionally characterized as proficient actions of (re)-generating a representation to express one's thinking and appropriately reading-out from a representation. However, as we've have demonstrated, inscriptions can provide more than a place to read-out information, but can be a site for action. Additionally, there is a notion of proficient representation use involving looking through the representation to the referent. In some places, Chad's sense-making empirically lacks a physical referent, and is based solely on the figural form of the inscription. Although the physical referent may be present in Chad's internal thinking, it need not be for him to come up with his final representation.

\section{ACKNOWLEDGEMENTS}

The authors thank the UMD PERG, Noah Finkelstein, and Jessica Hoehn. Supported by NSF-DUE1323129.

[1] P.B. Kohl, D. Rosengrant, \& N.D. Finkelstein, J. Learn Sci., 19, 3 (2007).

[2] S. Ainsworth, Comp \& Ed., 33, 131 (1999).

[3] P. Heller, M. Hollabaugh, Am. J. Phys. 60, 637 (1992).

[4] T. Fredlund, J. Airey, \& C. Linder, Euro. J. of Phys, 33, 657 (2012).

[5] P.B. Kohl, D. Rosengrant, \& N.D. Finkelstein, Phys. Rev., 1, 1 (2005).

[6] L.C. McDermott, Am. J. Phys, 55, 503 (1987).

[7] S. McKagan \& C. Wieman, AIP Con. Pro., 818, 2006).

[8] A.F. Heckler, Int J. Learn Sci., 32, 1829 (2010).

[9] E. Gire, Phys. Rev., 11, 1 (2015).

[10] O. Parnafes J. Learn Sci., 16, 415 (2007).

[11] J. Greeno \& R.Hall, Phi Delta Kappa, 78, 361 (1997).

[12] O. Parnafes and A. Disessa, Hum. Dev. 56, 5 (2013).

[13] P. Cobb, E. Yackel,\& K. McClain, Symbolizing and

communicating in mathematics classroom, 325. (2000).

[14] S. Derry et al, J. Learn Sci. 19, 3 (2010).

[15] R.S. Russ, R.E. Scherr, D. Hammer, \& J. Mikeska,

Sci. Ed., 92, 499 (2008).

[16] C. Goodwin, Am. Anthropol., 96, 606 (1994). 